OPEN ACCESS

Edited by:

Pietro Quaglino,

University of Turin, Italy

Reviewed by:

Kim Allyson Margolin, John Wayne Cancer Institute,

United States

Xiuli Wang,

Tongji University School of Medicine,

China

Peiru Wang,

Shanghai Skin Disease Hospital, China

*Correspondence:

Paolo Bossi

paolo.bossi@unibs.it

Specialty section:

This article was submitted to

Skin Cancer,

a section of the journal

Frontiers in Oncology

Received: 17 June 2021 Accepted: 03 December 2021 Published: 05 January 2022

Citation:

Alberti $A$ and Bossi P (2022)

Immunotherapy for Cutaneous

Squamous Cell Carcinoma:

Results and Perspectives.

Front. Oncol. 11:727027.

doi: 10.3389/fonc.2021.727027

\section{Immunotherapy for Cutaneous Squamous Cell Carcinoma: Results and Perspectives}

\author{
Andrea Alberti and Paolo Bossi ${ }^{*}$ \\ Medical Oncology, Department of Medical and Surgical Specialties, Radiological Sciences and Public Health University of \\ Brescia, Azienda Socio Sanitaria Territoriale (ASST)-Spedali Civili, Brescia, Italy
}

Although initial surgical excision cures $95 \%$ of patients, a minority of cutaneous squamous cell carcinomas (CSCCs) are judged to be unresectable, either locally advanced or with unresectable regional lymph nodes or distant metastases. These patients are offered systemic treatments. Response rate to chemotherapy is relatively low and not durable, as well as the results obtained with epidermal growth factor inhibitors (EGFRi). Like other cutaneous tumors, cSCCs have high immunogenicity, driven by the high mutational burden, the ultraviolet signature, and the overexpressed tumor antigens. Two checkpoint inhibitors, cemiplimab and pembrolizumab, achieved high response rate and survival with fewer toxicities than other available systemic agents. These promising results prompted to investigate new combination strategies of systemic therapy and surgery or radiotherapy. Subgroup analysis showed promising role of immunotherapy to facilitate surgery in locally advanced cSCC and, in a small group of patients, long-term survivals without resection. However, some cSCCs treated with immunotherapy develop either early or late resistance, so new drugs and new combinations are in a clinical study to overcome the mechanism underpinning these resistances. The present review focuses on the progress with immunotherapy to date and on new therapeutic strategies for cSCC.

Keywords: cutaneous squamous cell carcinoma, immunocheck point inhibitors, neoadjuvan, adjuvant, transplantation, future perspective

\section{INTRODUCTION}

Cutaneous squamous cell carcinoma ( $\mathrm{CSCC}$ ) is the second most common non-melanoma skin cancer and accounts for $20 \%$ of all deaths from skin cancer $(1,2)$. The estimated incidence of new cSCC cases in the UK is between 15 and 35 per 100,000 people and is increasing (3). The vast majority of patients have a limited disease, so can be successfully managed with a variety of simple procedures, such as cryotherapy and curettage, topical treatments (fluorouracil, imiquimod), or simple surgical excision. When the lesions are more advanced, Mohs micrographic surgery, more extensive surgical resection, or radiotherapy or their combinations are generally sufficient to control the locoregional disease. Only 5\% of cSCCs are unresectable, locally advanced, or with nonresectable regional lymph nodes or distant metastases. This quote of patients represents the indication for systemic treatments. 
Only limited data are available on the role of systemic chemotherapy in the treatment of advanced cSCC. Cisplatin-based combinations appear to be the most active regimens $(4,5)$ and have been adapted from those used for SCC occurring at other noncutaneous sites. Based on non-randomized trials, systemic chemotherapy is able to achieve partial response (PR) in about 34$44 \%$ of the cases, with median progression-free survival (PFS) and overall survival (OS) of about 5 and 11 months, respectively $(5,6)$.

The epidermal growth factor receptor (EGFR) is highly expressed in many epithelial tumors. Although its tumoral expression is inversely correlated with clinical outcome (7), the degree of overexpression does not appear to correlate with the efficacy of EGFR inhibitors (8). In prospective studies on EGFR inhibition with antibodies or small molecules in patients with advanced cSCC, an objective response was reported in $10-31 \%$ of patients, and the median time of OS was 11-13 months (8-12). A phase 2 study on cetuximab reported an objective response of $28 \%$ and a mean OS of 8.1 months (10). Therefore, advanced CSCC is a life-threatening condition for patients treated with cytotoxic chemotherapy or EGFR inhibitors and is associated with substantial morbidity, quality of life impact, and health care burden. Patients over 65 years of age are more likely than younger patients to require dose reductions in the first cycle of chemotherapy, emphasizing the need for new therapeutic approaches in a predominantly elderly population $(13,14)$.

\section{WHY ARE CUTANEOUS SQUAMOUS CELL CARCINOMA SO IMMUNOGENIC?}

Long-term sun exposure leading to DNA damage is postulated to account for the high mutational burden, approximately 45 mutations per megabase (15-18). Furthermore, tumor suppressor genes are most frequently altered, with the UV signature being a key mutational difference (15). Because UV have a relevant role in an early phase of cutaneous cancers pathogenesis, several molecular studies demonstrated that cSCC has a high mutational burden, which likely results in higher levels of tumor neoantigens that may be targets for the immune system. Additionally, the strong link between immunosuppression and the risk of cutaneous squamous cell carcinoma (19) indicates that natural immunosurveillance has a strong role in controlling this tumor type. There are several posited mechanisms for immunosurveillance escape, in cSCC the more relevant being the promotion of an immune-tolerant microenvironment (20). This happens by manipulation of cytokines (increased secretion of IL-6, IL-10, and TGF-beta; consumption of IL-2) that encourages infiltration of Treg cells, myeloid-derived suppressor cells (MDSCs), and other cell types that negatively modulate immune response. These cells can then actively suppress proliferation of CD4+ and CD8+ $\mathrm{T}$ lymphocytes that would otherwise recognize tumor antigens $(20,21)$. cSCC also upregulates the expression of immune checkpoint molecules such as $\mathrm{PD}$ ligand 1 (PD-L1) that promote peripheral $\mathrm{T}$ cell exhaustion (22).

On the basis of these preclinical data, immune checkpoint inhibitors were tested in advanced cSCC with good results.

\section{CEMIPLIMAB AND PEMBROLIZUMAB FOR LOCOREGIONALLY ADVANCED OR METASTATIC DISEASE}

Cemiplimab and pembrolizumab are fully human monoclonal antibodies belonging to the class of immunoglobulin G4 (IgG4) class, which binds to the PD-1 and blocks its interaction with its ligands PD-L1 and PD-L2. The involvement of PD-1 with its PDL1 and PD-L2 ligands, which are expressed by antigenpresenting cells and may be expressed by tumor cells and/or other cells in the tumor microenvironment, results in inhibition of the T-cell function, such as proliferation, cytokine secretion, and cytotoxic activity.

Cemiplimab efficacy was investigated in cSCC in two expansion cohorts of phase I multicohort study ( $\mathrm{n}=26$ patients) $(23,24)$, and then in a phase II EMPOWER-CSCC 1 study ( $n=193$ patients) (25-29); both trials had an open-label, multicenter design. The phase I clinical trial demonstrated the safety and the activity of cemiplimab in cSCC. The response rate, as assessed by independent central review, was 50\% [95\% confidence interval (CI), 30 to 70 ] with a duration of response that exceeded 6 months in 7 of the 13 responding patients (23). These data have been confirmed across the three parallel treatment groups of phase II clinical trial [i.e., $3 \mathrm{mg} / \mathrm{kg}$ once every 2 weeks in Groups 1 (mcSCC) and 2 (lacSCC); $350 \mathrm{mg}$ once every 3 weeks in group 3 (mcSCC)]. Prior systemic treatment for cancer had been received by $33.7 \%$ of patients, while $90.2 \%$ of patients had previously had surgery for their cancer and $67.9 \%$ had received prior radiotherapy (30). An objective response was observed in 49.2, 43.6, and $41.1 \%$ of patients, in groups 1,2 , and 3 , respectively; with a median time to response of 1.9 months in groups 1 and 2 and 2.1 months in group $3(25,26)$. The response seen with cemiplimab in patients with advanced cSCC appeared to be durable; at the interim analyses, the median duration of response (DOR) and median survival was not reached, after a median follow-up of 16.5, 8.1, and 9.3 months for groups 1,3 , and 2 , respectively $(25,26)$. In mcSCC cohort patients with a DOR $\geq 12$ months was 22 of 29 responders (26) and in lacSCC patients 12 of 34 responders (25). Median OS and PFS were yet to be reached by any treatment group.

Across cSCC, pembrolizumab was investigated in two phase II trials, the KEYNOTE 629 ( $\mathrm{n}=105$ patients) and CARSKIN trial $(n=57$ patients in expansion cohort); both trials were open-label, single-arm, multicenter design. In KEYNOTE 629, majority of the patients had received one or more prior systemic therapies (87\%) or RT $(74 \%)$ (31). In the entire study population, the objective response rate was $34 \%$, with complete and partial response rates reported in 4 and 31\%, respectively. Among the cohort of 36 patients with confirmed disease response, approximately twothirds (69\%) experienced durable responses longer than 6 months. At a median follow-up of approximately 10 months, median PFS was 7 months, and 1-year OS was 60\% (31).

In the investigated initiated CARSKIN trial, where only treatment-naïve patients were enrolled, the objective response rate in the entire study population was $42 \%$, with a complete and 
partial response rate of 7 and 35\%, respectively (32). In the expansion cohort, the objective response rate was higher among those with programmed cell death ligand 1 (PD-L1)-positive disease (55\%) versus $\mathrm{PD}-\mathrm{L} 1$-negative disease (17\%) $(\mathrm{P}=0.02)$ (32). In the primary cohort, after a median follow-up of 22.4 months, any of 16 responders experienced a subsequent disease progression. In this cohort, the median PFS and OS were 7 and 25 months, respectively (32) (See Table 1).

Based on these results, even if the lack of major evidence of phase 3 trials and in the absence of a direct comparison between chemotherapy and immunotherapy, FDA and EMA approved cemiplimab and pembrolizumab for the treatment of advanced and metastatic CSCC. NCCN and ESMO guidelines recommend them as first-line therapy.

\section{SAFETY AND ADVERSE EVENTS OF IMMUNOTHERAPY IN ADVANCED AND METASTATIC CSCC}

Because of the advanced age and comorbidities in patients with cSCC, safety is one of the most important challenges. Across the above-presented trials, most treatment-related adverse events (TRAEs) were G1 to 2 , and only $13-19 \%$ were $G \geq 3$. Less than
$10 \%$ of patients discontinued the treatment because of toxicities, with a low number of treatment-related deaths $(25-27,31,32)$. Investigators distinguished "treatment-related adverse events (TRAE)" from "immune-related adverse events (irAE)," the second one linked to the probable immune-pathogenesis. Most immunemediated AEs were G1 or 2 and non-serious. In KN629, the most frequently reported ones were hypothyroidism $(8.8 \%)$, pneumonitis (3.8\%), hyperthyroidism (3.1\%), and severe skin reactions (3.1\%), while there were no grade 4 to 5 irAEs (31) (See Table 2).

We may therefore conclude that in advanced and metastatic cSCC, treatment with both cemiplimab and pembrolizumab is safe, with a spectrum of toxicities similar to what observed in other solid tumors. However, because comorbidities in patients with cSCC may be high, any added toxicities impact on patient's frailty, therefore suggesting the importance of an early recognition and treatment of immune-mediated AEs.

\section{IMMUNOTHERAPY IN ALLOGENIC ORGAN TRANSPLANTATION}

It is well-known that the state of immune tolerance induced by broad immunosuppression to prevent allograft rejection leads to an increased risk of the development of cSCC.

TABLE 1 | Overview of results from Keynote 629, Carskin and EMPOWER trial.

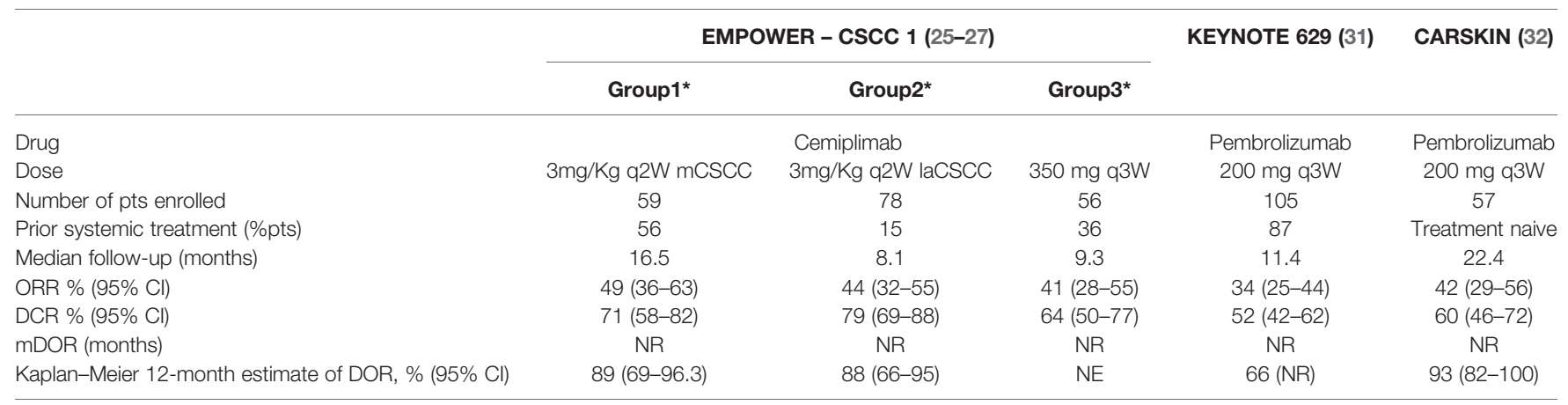

* Group 1, 3 mg/kg once every 2 weeks mCSCC; Group 2, 3 mg/kg once every 2 weeks laCSCC; Group 3, 350 mg once every 3 weeks.

CSCC, cutaneous squamous cell carcinoma; laCSCC, local advanced CSCC; $m C S C C$, metastatic CSCC; ORR, overall response rate; DCR, disease control rate; mDOR, median duration of response; $N R$, not reached; $N E$, not explain.

TABLE 2 | Overview of adverse events from Keynote 629, Carskin and EMPOWER trial.

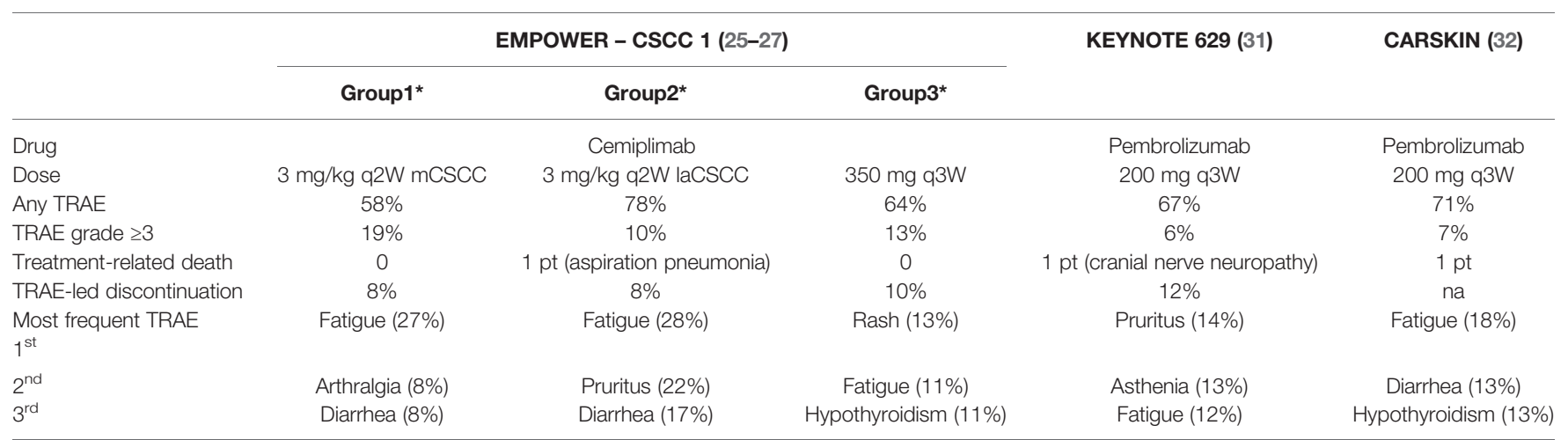

${ }^{*}$ Group 1, 3 mg/kg once every 2 weeks mCSCC; Group 2, 3 mg/kg once every 2 weeks laCSCC; Group 3, 350 mg once every 3 weeks.

CSCC, cutaneous squamous cell carcinoma; laCSCC, local advanced CSCC; mCSCC metastatic CSCC; TRAE, treatment related adverse events. 
Both CTLA-4 and PD-1/PD-L1 play a key role in immunotolerance required for allograft survival $(33,34)$. In a preclinical study, the injection of anti-CTLA-4 immunoglobulin in the perioperative period led to the acute rejection of liver allograft but did not have any effect on graft survival when it was injected after the establishment of peripheral tolerance (33). On the contrary, the early infusion of anti-PD-1 antibodies prevented the induction of peripheral tolerance, and infusion at a later stage led to the complete loss of allograft (33-35). Although this has not been proved in humans, several reports in literature are warning about the high rates (quite $40 \%$ ) of allograft rejection in patients with cancer who were treated with an ICI leading to organ failure in $71 \%$ of the patients who experienced rejection (36). The majority of graft rejections happened after 1-2 doses of ICIs, although no one has demonstrated that the loss of immunotolerance secondary to ICI is dose- and time-independent.

Accordingly, prospective studies using ICIs in organtransplanted patients with cancer are needed. The only prospective study reported to date is a small phase I clinical trial (37) testing the safety of nivolumab in four renal transplant recipients with multiple myeloma, head and neck SCC, renal cell carcinoma, and bladder cancer. The patients were required to have no human leukocyte antigen donor-specific antibodies (DSAs). Patients received one, two, three, and nine doses of nivolumab, respectively. None of the patients had a graft rejection, and only one patient (who received nine doses) experienced a partial response. Another phase I trial (38) is open and accruing patients with renal transplants diagnosed with unresectable or metastatic cutaneous carcinoma (melanoma-cSCC-basal cell carcinoma or Merkel cell carcinoma) to receive prednisone, tacrolimus, and nivolumab with the addition of ipilimumab upon the progression of the disease. The primary endpoint of the study is response rate. As of today, because of the high risk of allograft loss and the poor data of clinical benefit, the use of ICI should be discussed with patients clearly before the initiation of treatment, and these patients should be closely monitored for signs of rejection.

\section{FUTURE STRATEGIES: ADJUVANT AND NEOADJUVANT IMMUNOTHERAPY IN CSCC}

Clinical trials in melanoma showed that in the advanced metastatic treatment setting, patients with lower tumor burdens were more likely to experience long-term survival after anti-PD-1 therapy $(39,40)$. This suggests that postoperative (adjuvant) anti-PD-1 therapy directed against residual micrometastatic disease might prolong RFS and OS. The same impact in survival could be foreseen by anticipating immunotherapy before surgery (neoadjuvant), where using PDL1 blockade with primary tumor in place could leverage higher levels of endogenous tumor antigen to enhance T-cell priming.

In fact, anti-PD-(L) 1 rejuvenates tumor-specific cytotoxic $\mathrm{T}$ cells that already reside in the tumor microenvironment (TME), causing their activation, proliferation, and trafficking to micrometastatic deposits. Moreover, having tumor-draining lymph nodes (TDLN) in place could increase the antigen presentation by dendritic cell to T cells. Recently, Yost, Chang, and colleagues showed that after anti-PD-1 therapy of cutaneous squamous cell carcinomas, $\mathrm{T}$ cell clonal expansion was due to new clones "appearing" in the TME rather than expansion of clones already in the tumor before initiation of anti-PD-1 therapy; these findings suggest that other clones not present initially in the tumor traffic in it upon anti-PD-1 treatment (41). The melanoma trial by Blank et al. comparing neoadjuvant versus adjuvant regimens of anti-PD-1 plus anti-CTLA-4 found a greater expansion of tumor-resident $\mathrm{T}$-cell clones in the peripheral blood of patients enrolled on the neoadjuvant arm (42). These clones persisted in the periphery for weeks after tumor resection (41).

Based on this strong biological rationale and on data from melanoma studies, several ongoing trials are investigating the role of checkpoint inhibitors in neoadjuvant settings for patients with cSCC. They differ from each other at first for inclusion criteria, because they enroll only patients with high-risk tumor, but the risk of recurrence is established by different factors. To date, the most used reference to identify high-risk patients is the American Joint Committee on Cancer staging 8th edition (AJCC-8) (43). High-risk cSCCs are therefore defined according to tumor diameter, lymph node size, the number of positive lymph nodes and their location(s) (ipsilateral, contralateral, bilateral), and extranodal extension. However, the AJCC-8 is relevant only for head-and-neck cSCCs, which might limit its usefulness. Other risk factors are considered by the Brigham and Women's Hospital (BWH)-staging system (43), when having all of these characteristics: tumor diameter $\geq 2 \mathrm{~cm}$, tumor invasion beyond subcutaneous fat, perineural invasion $\geq 0.1 \mathrm{~mm}$ and poorly differentiated cSCC, or bone invasion.

After all, the correct selection of patient population in the neoadjuvant setting is an important challenge. Special considerations are centered on risk-benefit expectations in patient populations among which a proportion would be cured by surgery alone and on the other hand the risk of severe and prolonged immune-related adverse effects.

Another point of difference between trials in these settings is the primary endpoint used as surrogate of survival. Disease-free survival is the historical surrogate of overall survival in the adjuvant setting, although differences exist across neoadjuvant immunotherapy clinical studies. This is because the role of response rate as a surrogate of survival in this setting is not well established. Pathologic response criteria for neoadjuvant cancer therapy were first developed in the context of chemotherapy as a parameter portending clinical outcomes. Pathologic complete response (pCR), the most stringent criterion, is defined as the absence of any viable tumor in the definitive surgical resection specimen. To date, $\mathrm{pCR}$ and major pathologic response (MPR) defined as describing a treatment effect resulting in $\leq 10 \%$ residual viable tumor are the most commonly used metrics for assessing response to neoadjuvant immunotherapy, although differences exist across clinical studies. 
Similar to the precedent established with non-immunologic neoadjuvant therapies, the degree of pathologic response may help assign patients to postsurgical observation or intervention, and ongoing studies will answer this question.

If these new therapeutic strategies would translate into a clinical benefit, new questions will arise regarding the type of surgery and the role of postoperative radiotherapy. At first on the surgical treatment, if an aggressive or cosmetically disfiguring surgery would have been recommended in the absence of neoadjuvant therapy, would the same surgical approach still be required for tumors exhibiting a major response to neoadjuvant treatment? Limited surgical interventions could be used in patients whose on-treatment tumor biopsies show a complete or major pathologic response, for example, as provided in melanoma in an extension cohort of NCT02977052.

\section{FUTURE STRATEGIES: IMMUNOTHERAPY CONCOMITANT TO RADIATION}

Another innovative strategy in cSCC therapy is the combination of immunotherapy with radiotherapy. The discovery that radiation-induced damage to tumor tissues and normal tissues in the radiation field can trigger the activation of the immune system via well-known damage-signaling cascades, immunogenic cell death, or both has led to a paradigm change in the use of radiotherapy. Preclinical and clinical investigations revealed a complex interplay between radiotherapy, irradiated cells and tissues, and the immune system (44-46); for example, exposure to radiotherapy was shown to upregulate major histocompatibility complex I (MHC I) expression in tumor cells, modulate immunosuppressive barriers in the tumor microenvironment, activate restrictive tumor vessels, trigger the recruitment of immune effector cells to the local tumor, and even elicit systemic tumor-specific immune responses (47, 48). The efficacy of synergy between radiotherapy and checkpoint inhibitors in squamous cell carcinoma of the skin is being studied in the UNSCARRed trial: a single-arm, interventional study combining avelumab with radical radiotherapy, accrual is ongoing.

\section{NEW IMMUNOTHERAPY DRUGS AND COMBINATIONS}

Increased understanding of the underlying immunologic mechanisms is leading to the identification of several additional potential targets to unleash the immune system and control malignancy. These approaches include new checkpoint inhibitors, agonist of costimulatory receptors, manipulation of $\mathrm{T}$ cells, oncolytic viruses, cytokines, and vaccines. In addition to implement the response to immunotherapy and increase survival, new pharmacological combinations are under investigation.

\section{New Checkpoint Inhibitors and Combinations}

Data on anti-CTLA4 (e.g., Ipilimumab or tremelimumab) for cSCC are limited; their association with anti-PD1 is still in study in the neoadjuvant setting (NCT04620200) and in allograft patients (NCT03816332) (38).

TIGIT is another inhibitory receptor co-expressed with PD-1 on tumor antigen-specific CD8+ T cells and CD8+ tumorinfiltrating lymphocytes (TILs). It is highly expressed by Tregs in peripheral blood mononuclear cells of healthy donors and patients with cancer and further upregulated in the TME (49). Promising data on anti-TIGIT monoclonal antibody Tiragolumab efficacy in NSCLC had been presented at ASCO virtual meeting 2020 (50). As far as we know, there are no trials ongoing with this drug in cSCC. CD47 is another promising target. It is upregulated in essentially every cancer type to inhibit innate immune cells from phagocytosing the tumor cells. A humanized anti-CD47 monoclonal antibody demonstrated promising results in non-Hodgkin lymphoma (51), and the evaluation of the activity in CSCC is ongoing (NCT04502888).

Another therapeutic strategy, formulated to increase the response rate of immunotherapy and to overcome mechanisms of resistance to progression, is the addition of an anti-EGFR agent. The hypothesis of I-TACKLE (NCT03666325) trial is that the adjunct of an anti EGFR agent as cetuximab could reverse the primary and secondary resistance to pembrolizumab, with a synergistic effect able to counteract pathway redundancy (i.e., the presence of several concurrent pathways which need to be addressed together) and boosting T-cell priming. Enrollment is underway, and the results of a first analysis are expected soon. Another trial with avelumab concurrently with cetuximab is currently ongoing (NCT03944941).

Checkpoint inhibitors and modulators of DNA damage response (DDR) is another pharmacological association being studied in several neoplasms. PARPi-mediated catastrophic DNA damage is a favorable factor for ICI therapy, and the relationship between tumor mutation burden and efficacy of ICI has been confirmed in previous studies (52). Apart from tumor mutation burden, DDR-mediated immune responses collaborate with ICI, which remodel tumor immune microenvironment and boost the cancer immunity cycle (53). In this way, PARPimediated acute inflammation remodels tumor immune microenvironment and drives a systemic Th1-skewing immune response. In cSCC, a trial is ongoing with pembrolizumab plus abexinostat (NCT03590054), a pan-histone deacetylase inhibitor and inhibitor of RAD51, which is involved in repairing DNA double-strand breaks.

\section{Agonists of Costimulatory Receptors}

Multiple costimulatory receptors are involved in the immune response to tumors and hence are potential targets for cancer immunotherapy. Inducible T-cell costimulator (ICOS), CD40, CD28 agonists are only some examples of costimulatory receptors, studied in preclinical animal models and some of them also in early phases of clinical development (54). CD40 has been identified as an interesting immunotherapy target in 
human cancers by virtue of its ability to stimulate helper T-cell immune response and macrophage differentiation (55). CD40 ligand (CD40L) gene therapy has been shown to increase tumorinfiltrating $\mathrm{T}$ cells in vivo and demonstrated an oncolytic effect. SL-172154 is an engineered monoclonal antibody that consists of Sirp $\alpha$ linked to CD40L, providing checkpoint blockade (CD47 axis) and potent costimulation (CD40 axis). By blocking this signal through Sirp $\alpha$ binding, the surface of the tumor cells is coated with the drug, allowing the CD40L side to bind to CD40 on APCs, which will lead to enhanced antigen presentation to CD8+ and CD4+ T lymphocytes and tumor cell phagocytosis. The trial with SL-172154 in cSCC is ongoing (NCT04502888).

\section{Cytokines}

Initial approaches to immunotherapy harnessed the numerous downstream effects of cytokines. IL2 and INFa demonstrate mild efficacy in melanoma and renal cell carcinoma. Other interleukin analogs or interleukin receptor agonists have been studied in the preclinical setting, with poor results. Today promising results are expected by the combination between atezolizumab and NT-I7 (recombinant human IL-7-hybrid Fc), which acts through IL-7 receptor (IL-7R), which is expressed on naïve and memory CD4+ and CD8+ T cells. Thus, IL-7 promotes proliferation, maintenance, and functionality of these key T-cell subsets mediating immune responses. The trial with this association in cSCC is ongoing (NCT03901573).

\section{Oncolytic Viruses}

Oncolytic viruses mediate antitumor effects in several ways. Viruses can be engineered to efficiently infect cancer cells preferentially over normal cells, to promote the presentation of tumor-associated antigens, to activate signals that promote a less immune-tolerant tumor microenvironment, and to serve as transduction vehicles for the expression of immunemodulatory cytokines (56). Injection of oncolytic viruses may synergize with checkpoint inhibitors by increasing CD8+ T cell infiltration and IFN gamma signaling as well as upregulating PD-

L1 in the microenvironment (57).

RP1 is an oncolytic HSV that encodes a fusogenic GALV-GP $\mathrm{R}$ - protein and granulocyte-macrophage colony-stimulating factor (GM-CSF). RP1 demonstrated tolerable safety and tumor regression alone and with nivolumab in patients with several tumor types, including cSCC (57). Another promising oncolytic virus is Talimogene laherparepvec (TVEC). It is again an HSV-1, modified to lose the neurovirulence and include the capacity to express GM-CSF. This allows for preferential replication within tumor cells resulting in cell lysis. Additionally, the release of virally derived GM-CSF along with antigens derived from ruptured tumor cells can induce a systemic tumor-specific immune response which may lead to regression of distant uninjected lesions (58).

\section{Vaccines}

Many attempts have been made to harness the adaptive immune recognition of a cancer-related antigen to effect antitumor responses. There are many methods of vaccination, and the choice of antigen, schedules of administration, and adjuvants can influence an adaptive immune response. Antigen choices range from simple peptides, which are easy to administer but affect a narrow antigen spectrum and are often restricted by specific HLA class 1, to whole-cell preparations that offer a broader range of antigens but are more costly and time-consuming to prepare (59). Given the increasing understanding of the importance of immune recognition of not only multiple tumor antigens but specific ones for each patient, current studies to develop therapeutic vaccines are beginning to explore the use of individualized pooled antigens. Several efforts on these strategies are being made in cSCC.

Other drugs with alternative mechanisms of action, external to the immune synapse, are under investigation. Among the molecular ones suitable to stimulate antitumor immune effects, a toll-like receptor 9 (TLR9) agonist, CMP-001, activates plasmacytoid dendritic cells (pDCs) and triggers interferon alpha (IFN $\alpha$ ) release. These lead to a cascade of antitumor immune effects. Ongoing trials are testing CMP-001 in combination with checkpoint inhibitors in several solid tumors, also in cSCC (NCT03684785).

\section{CONCLUSIONS}

The treatment of locally advanced metastatic cSCC remains a challenge. Chemotherapy may achieve response rata in about onethird of the patients, as targeted agents against EGFR $(4,5,10)$; however, the main limitations of these approaches are the relatively short duration of response and the adverse events, often not compatible with the frailty of the patients. In contrast, immune checkpoint inhibitors showed a high response rate, a long duration of response, and a better toxicity profile $(25-27,31,32)$. This has broadened the therapeutic armamentarium to be offered to cSCC patients and the possibility to treat also elderly patients with comorbidities otherwise not amenable to systemic treatments. However, new questions arise from this new approach. First of all, the search for predictive factors of response to treatment remains an unresolved challenge, with no molecular or clinical factor able to identify patients with a greater possibility of obtaining a benefit from immunotherapy. Moreover, it is not clear up to now how long to continue the treatment in patients who achieve a complete response with immune checkpoint inhibitors. The role of immunotherapy as adjuvant or neoadjuvant treatment is being investigated in ongoing clinical trials, thus representing a new frontier for multidisciplinary approaches. Behind this, if immunotherapy has proven to be an effective strategy, new drug combinations with novel mechanisms of actions are being investigated to improve the results till now considered and to overcome the resistance mechanisms.

\section{AUTHOR CONTRIBUTIONS}

PB contributed to conception of the study. AA wrote the first draft of the manuscript. PB and AA contributed to manuscript revision, read, and approved the submitted version. 


\section{REFERENCES}

1. Rogers HW, Weinstock MA, Feldman SR, Coldiron BM. Incidence Estimate of Nonmelanoma Skin Cancer (Keratinocyte Carcinomas) in the U.S. Population, 2012. JAMA Dermatol (2015) 151:1081-6. doi: 10.1001/ jamadermatol.2015.1187

2. Gurney B, Newlands C. Management of Regional Metastatic Disease in Head and Neck Cutaneous Malignancy. Cutaneous Squamous Cell Carcinoma. $\mathrm{Br} \mathrm{J}$ Oral Maxillofac Surg (2014) 52:294-300. 3. doi: 10.1016/j.bjoms.2014.01.015

3. Burton KA, Ashack KA, Khachemoune A. Cutaneous squamous cell carcinoma: A review of high-risk and metastatic disease. Am J Clin Dermatol (2016) 17:491-508. doi: 10.1007/s40257-016-0207-3

4. Sadek H, Azli N, Wendling JL, Cvitkovic E, Rahal M, Mamelle G, et al. Treatment of Advanced Squamous Cell Carcinoma of the Skin With Cisplatin, 5-Fluorouracil, and Bleomycin. Cancer (1990) 66(8):1692-6. doi: 10.1002/1097-0142(19901015)66:8<1692::AID-CNCR2820660807>3.0.CO;2-Y

5. Karkiwski A, Hare R, Loud P, Skitzki J, Kane J, May K, et al. Systemic Therapy in Advanced Cutaneous Squamous Cell Carcinoma (CSCC): The Roswell Park Experience and a Review of the Literature. Am J Clin Oncol (2016) 39:545. doi: 10.1097/COC.0000000000000088

6. Fitzgerald K, Tsai KK. Systemic Therapy for Advanced Cutaneous Squamous Cell Carcinoma Semin Cutan. Med Surg (2019) 38(1):E67-74. doi: 10.12788/ j.sder.2019.010

7. Randis JR, Zeng Q, Drenning SD, Tweardy DJ. Normalization of EGFR Mrnalevels Following Restoration of Wild-Type P53 in a Head and Neck Squamous Cellcarcinoma Cell Line. Int J Oncol (1998) 13:375-8. doi: 10.3892/ ijo.13.2.375

8. Foote MC, McGrath M, Guminski A, Hughes BGM, Meakin J, Thomson D, et al. Phase II Study of Single-Agent Panitumumab in Patients With Incurable Cutaneous Squamous Cell Carcinoma. Ann Oncol (2014) 25:2047-52.7. doi: 10.1093/annonc/mdu368

9. William WN Jr, Feng L, Ferrarotto R, Ginsberg L, Kies M, Lippman S, et al. Gefitinib for Patients With Incurable Cutaneous Squamous Cell Carcinoma: A Single-Arm Phase II Clinical Trial. J Am Acad Dermatol (2017) 77:111013.e2. doi: 10.1016/j.jaad.2017.07.048

10. Maubec E, Petrow P, Scheer-Senyarich I, Duvillard P, Lacroix L, Gelly J, Certain A, et al. Phase II Study of Cetuximab as First-Line Single-Drug Therapy in Patients With Unresectable Squamous Cell Carcinoma of the Skin. J Clin Oncol (2011) 29:3419-26. doi: 10.1200/JCO.2010.34.1735

11. Cavalieri S, Perrone F, Miceli R, Ascierto PA, Locati LD, Bergamini C, et al. Efficacy and Safety of Singleagent Pan-Human Epidermal Growth Factor Receptor (HER) Inhibitor Dacomitinib in Locally Advanced Unresectable or Metastatic Skin Squamous Cell Cancer. Eur J Cancer (2018) 97:7-15. doi: 10.1016/j.ejca.2018.04.004

12. Gold KA, Kies MS, William WN Jr, Johnson FM, Lee JJ, Glisson BS. Erlotinib in the Treatment of Recurrent or Metastatic Cutaneous Squamous Cell Carcinoma: A Single-Arm Phase 2 Clinical Trial. Cancer (2018) 124:216973. doi: $10.1002 /$ cncr.31346

13. Cowey C, Robert N, Davies K, Davies K, Frytak F, Lowy I, et al. Treatment Patterns and Outcomes Among Patients With Advanced Cutaneous Squamous Cell Carcinoma (CSCC) in a US Community Oncology Setting. Proc Am Soc Clin Oncol (2019) 37:e21033. doi: 10.1200/JCO.2019.37.15_suppl.e21033

14. Ruiz E, Chen CI, Deering K. Treatment Patterns and Costs in Cutaneous Squamous Cell Carcinoma (CSCC) Patients With Nodal Dissection, Chemotherapy, and/or Radiation Therapy. Proc Am Soc Clin Oncol (2018) 36:e18703 (abstr). doi: 10.1200/JCO.2018.36.15_suppl.e18703

15. Pickering CR, Zhou JH, Lee JJ, Drummond LA, Peng SA, Saade RE, et al. Mutational Landscape of Aggressive Cutaneous Squamous Cell Carcinoma. Clin Cancer Res (2014) 20:6582-92. doi: 10.1158/1078-0432.CCR-14-1768

16. Kim Y, He YY. Ultraviolet Radiation-Induced Non-Melanoma Skin Cancer: Regulation of DNA Damage Repair and Inflammation. Genes Dis (2014) 1:188-98. doi: 10.1016/j.gendis.2014.08.005

17. Madan V, Lear JT, Szeimies RM. Non-Melanoma Skin Cancer. Lancet (2010) 375:673-85. doi: 10.1016/S0140-6736(09)61196-X

18. Chalmers ZR, Connelly CF, Fabrizio D, Gay L, Ali SM, Ennis R, et al. Analysis of 100000 Human Cancer Genomes Reveals the Landscape of Tumor Mutational Burden. Genome Med (2017) 9:34. doi: 10.1186/s13073-0170424-2
19. Buttner R, Longshore JW, Lopez-Rios F, Merkelbach-Bruse S, Normanno N, Rouleau E, et al. Implementing TMB Measurement in Clinical Practice: Considerations on Assay Requirements. ESMO Open (2019) 4:e000442. doi: 10.1136/esmoopen-2018-000442

20. Lai C, August S, Albibas A, Behar R, Cho SY, Polak M, et al. Ox40+ Regulatory T Cells in Cutaneous Squamous Cell Carcinoma Suppress Effector T-Cell Responses and Associate With Metastatic Potential. Clin Cancer Res (2016) 22:4236-48. doi: 10.1158/1078-0432.CCR-15-2614

21. Bluth MJ, Zaba LC, Moussai D, Suarez-Farinas M, Kaporis H, Fan L, et al. Myeloid Dendritic Cells From Human Cutaneous Squamous Cell Carcinoma Are Poor Stimulators of T-Cell Proliferation. I Invest Dermatol (2009) 129:2451-62. doi: 10.1038/jid.2009.96

22. Slater NA, Googe PB. PD-L1 expression in cutaneous squamous cell carcinoma correlates with risk of metastasis. J Cutan Pathol (2016) 43:66370. doi: $10.1111 /$ cup. 12728

23. Migden MR, Rischin D, Schmults CD. PD-1 Blockade With Cemiplimab in Advanced Cutaneous Squamous-Cell Carcinoma. N Engl J Med (2018) 379:341-51. doi: 10.1056/NEJMoa1805131

24. Owonikoko TK, Papadopoulos KP, Johnson ML, Martin MG, Moreno V, Salama AKS, et al. Phase I Study of Cemiplimab, a Human Monoclonal AntiPD-1, in Patients With Unresectable Locally Advanced or Metastatic Cutaneous Squamous Cell Carcinoma (CSCC): Longer Follow-Up Efficacy and Safety Data [Abstract No 71P Plus Poster]. Ann Oncol (2018) 29(Suppl 10)::x25. doi: 10.25251/skin.2.supp.78

25. Migden MR, Khushalani NI, Chang ALS, Lewis KD, Schmults CD, Hernandez-Aya L. Cemiplimab in Locally Advanced Cutaneous Squamous Cell Carcinoma: Results From an Open-Label, Phase 2, Single-Arm Trial. Lancet Oncol (2020) 21:294-305. doi: 10.1016/S1470-2045(19)30728-4

26. Rischin D, Migden MR, Lim AM, Schmults C, Khushalani NI, Schadendorf D, et al. Phase 2 Study of Cemiplimab in Patients With Metastatic Cutaneous Squamous Cell Carcinoma: Primary Analysis of Fixed-Dosing, Long-Term Outcome of Weight-Based Dosing. J Immunother Cancer (2020) 8:e000775. doi: 10.1136/jitc-2020-000775

27. Migden MR, Khushalani NI, Chang ALS, Rischin C, Schmults CD, HernandezAya LF, et al. Primary Analysis of Phase 2 Results of Cemiplimab, a Human Monoclonal Anti-PD-1, in Patients With Locally Advanced Cutaneous Squamous Cell Carcinoma (laCSCC) [Abstract No. 6015 Plus Poster]. J Clin Oncol (2019) 37(15 Suppl):6015. doi: 10.1200/JCO.2019.37.15_suppl.6015

28. Rischin D, Lim AM, Schmults CD, Khushalani NI, Hughes BG, Schadendorf D, et al. Phase 2 Study of 2 Dosing Regimens of Cemiplimab, a Human Monoclonal Anti-PD-1, in Metastatic Cutaneous Squamous Cell Carcinoma (mCSCC) [Abstract Plus Poster]. Ann Oncol (2019) 30(suppl_5):v533-63. doi: 10.1093/annonc/mdz255.

29. Guminski AD, Lim AML, Khushalani NI, Schmults C, Hernandez-Aya L, Modi B, et al. Phase 2 Study of Cemiplimab, a Human Monoclonal Anti-PD-1, in Patients With Metastatic Cutaneous Squamous Cell Carcinoma (mCSCC; Group 1): 12-Month Follow-Up [Abstract No 9526 plus Poster] . J Clin Oncol (2019) 37(15 Suppl):9526. doi: 10.1200/JCO.2019.37.15_suppl.9526

30. Lee A, Duggan S, Deeks ED. Cemiplimab: A Review in Advanced Cutaneous Squamous Cell Carcinoma. Drugs (2020) 80:813-9. doi: 10.1007/s40265-02001302-2

31. Grob J-J, Gonzalez R, Basset-Seguin N, Vornicova O, Schachter J, Joshi A, et al. Pembrolizumab Monotherapy for Recurrent or Metastatic Cutaneous Squamous Cell Carcinoma: A Single-Arm Phase II Trial (KEYNOTE-629). J Clin Oncol (2020) 38: (25):2916-25. doi: 10.1200/JCO.19.03054

32. Maubec E, Boubaya M, Petrow P, Baylor-Barry M, Basset-Seguin N, Grob JJ, et al. Phase II Study of Pembrolizumab As First-Line, Single-Drug Therapy for Patients With Unresectable Cutaneous Squamous Cell Carcinomas. J Clin Oncol (2020) 38(26):3051-61. doi: 10.1200/JCO.19.03357

33. Judge TA, Wu Z, Zheng XG, Sharpe AH, Sayegh MH, Turka LA, et al. The Role of CD80, CD86, and CTLA4 in Alloimmune Responses and the Induction of Long-Term Allograft Survival. J Immunol (1999) 162:1947-51.

34. Li W, Zheng XX, Kuhr CS, Perkins JD. CTLA4 Engagement Is Required for Induction of Murine Liver Transplant Spontaneous Tolerance. Am J Transplant (2005) 5:978-86. doi: 10.1111/j.1600-6143.2005.00823.x

35. Aguirre LE, Guzman ME, Lopes G, Hurley J. Immune Checkpoint Inhibitors and the Risk of Allograft Rejection: A Comprehensive Analysis on an Emerging Issue. Oncol (2019) 24:394-401. doi: 10.1634/theoncologist.2018-0195 
36. Kumar V, Shinagare A b, Rennke H g, Ghai S, Lorch JH, Ott P, et al. The Safety and Efficacy of Checkpoint Inhibitors in Transplant Recipients: A Case Series and Systematic Review of Literature. Oncology (2020) 25:505-14. doi: 10.1634/theoncologist.2019-0659

37. Carroll R, Zalcberg J, Tang H. 1208p PD-1 Blockade in Renal Transplant Patients With Poor Prognosis Cancer and Minimizing Risk of Organ Rejection Using Comprehensive Immune Monitoring and Screening Techniques: A Safety Study. Ann Oncol (2018) 29(suppl 8):viii400. doi: 10.1093/annonc/mdy288

38. Tacrolimus, Nivolumab, and Ipilimumab in Treating Kidney Transplant Recipients With Selected Unresectable or Metastatic Cancers. Available at: https://clinicaltrials.gov/ct2/show/NCT03816332 (Accessed June 30, 2019).

39. Long GV, Atkinson V, Lo S, Sandhu S, Guminski AD, Brown MP, et al. Combination Nivolumab and Ipilimumab or Nivolumab Alone in Melanoma Brain Metastases: A Multicentre Randomised Phase 2 Study. Lancet Oncol (2018) 19(5):672-81. doi: 10.1016/S1470-2045(18)30139-6

40. Larkin J, Minor D, D’Angelo S, Neyns B, Smylie M, Miller WH, et al. Overall Survival in Patients With Advanced Melanoma Who Received Nivolumab Versus Investigator's Choice Chemotherapy in CheckMate 037: A Randomized, Controlled, Open-Label Phase III Trial. J Clin Oncol (2018) 36 (4):383-90. doi: 10.1200/JCO.2016.71.8023

41. Yost KE, Satpathy AT, Wells DK, Qi Y, et al. Clonal Replacement of TumorSpecific T Cells Following PD-1 Blockade. Nat Med (2019) 25:1251-9. doi: 10.1038/s41591-019-0522-3

42. Blank CU, et al. Neoadjuvant Versus Adjuvant Ipilimumab Plus Nivolumab in Macroscopic Stage III Melanoma. Nat Med (2018) 24:1655-61. doi: 10.1038/ s41591-018-0198-0

43. Stamell Ruiz E, Karia PS, Besaw R, Schmuls CD. Performance of the American Joint Committee on Cancer Staging Manual, 8th Edition vs the Brigham and Women's Hospital Tumor Classification System for Cutaneous Squamous Cell Carcinoma. JAMA Dermatol (2019) 155(7):819-25. doi: 10.1001/ jamadermatol.2019.0032

44. DeWolf K, Kruse V, Sundahl N, van Gele M, Chevolet I, Speeckaert R, et al. A Phase II Trial of Stereotactic Body Radiotherapy With Concurrent Anti-PD1 Treatment in Metastatic Melanoma: Evaluation of Clinical and Immunologic Response. J Transl Med (2017) 15:21. doi: 10.1186/s12967-017-1123-x

45. Roger A, Finet A, Boru B, Beauchet A, Mazeron JJ, Otzmeguine Y, et al. Efficacy of Combined Hypo-Fractionated Radiotherapy and Anti-PD-1 Monotherapy in Difficult-to-Treat Advanced Melanoma Patients. Oncoimmunology (2018) 7:e1442166. doi: 10.1080/2162402X.2018.1442166

46. Sharabi AB, Nirschl CJ, Kochel CM, Nirschl TR, Francica BJ, Velarde E, et al. Stereotactic Radiation Therapy Augments Antigen-Specific PD-1-Mediated Antitumor Immune Responses via Cross-Presentation of Tumor Antigen. Cancer Immunol Res (2015) 3:345-55. doi: 10.1158/2326-6066.CIR-14-0196

47. Twyman-Saint Victor C, Rech AJ, Maiy A, Rengan R, Pauken KE, Benci JL, et al. Radiation and Dual Checkpoint Blockade Activate Non-Redundant Immune Mechanisms in Cancer. Nature (2015) 520(7547):373-7. doi: 10.1038/nature14292

48. Reynders K, Illidge T, Siva S, Chang JY, De Ruysscher D. The Abscopal Effect of Local Radiotherapy: Using Immunotherapy to Make a Rare Event Clinically Relevant. Cancer Treat Rev (2015) 41(6):503-10. doi: 10.1016/j.ctrv.2015.03.011

49. Fourcade J, Sun Z, Chauvin J-M, Ka M, Davar D, Pagliano O, et al. Cd226 Opposes TIGIT to Disrupt Tregs in Melanoma. JCI Insight (2018) 3 (14):121157. doi: 10.1172/jci.insight.121157

50. Horvath L, Pircher A. ASCO 2020 Non-Small Lung Cancer (NSCLC) Personal Highlights. Memo (2021) 13:1-4. doi: 10.1007/s12254-020-00673-2

51. Advani R, Flinn I, Popplewell L, Forero A, Barlett NL, Ghosh N, et al. CD47 Blockade by Hu5F9-G4 and Rituximab in Non-Hodgkin's Lymphoma. N Engl J Med (2018) 379:1711. doi: 10.1056/NEJMoa1807315

52. Li A, Yi M, Qin S. Prospects for Combining Immune Checkpoint Blockade With PARP Inhibition. J Hematol Oncol (2019) 12:98. doi: 10.1186/s13045019-0784-8
53. Mouw KW, Goldberg MS, Konstantinopoulos PA, D’Andrea AD. DNA Damage and Repair Biomarkers of Immunotherapy Response. Cancer Discov (2017) 7:675-93. doi: 10.1158/2159-8290.CD-17-0226

54. van der Merwe PA, Dushek O. Mechanisms for T Cell Receptor Triggering. Nat Rev Immunol (2011) 11:47. doi: 10.1038/nri2887

55. Hassan SB, Sørensen JF, Olsen BN, Pedersen AE. Anti-CD40-Mediated Cancer Immunotherapy: An Update of Recent and Ongoing Clinical Trials. Immunopharmacol Immunotoxicol (2014) 36:96. doi: 10.3109/08923973. 2014.890626

56. de Gruijl TD, Janssen AB, van Beusechem VW. Arming Oncolytic Viruses to Leverage Antitumor Immunity. Expert Opin Biol Ther (2015) 15:959. doi: 10.1517/14712598.2015.1044433

57. Middleton MR, Aroldi F, Sacco J, et al. An Open-Label, Single-Arm, Phase II Clinical Trial of RP1, an Enhanced Potency Oncolytic Herpes Virus, Combined With Nivolumab in Four Solid Tumor Types: Initial Results From the Skin Cancer Cohorts. J Clin Oncol 38(15_suppl):e22050. doi: 10.1200/JCO.2020.38.15_suppl.e22050

58. Perez MC, Miura JT, Mahrukh Hussnain Naqvi S, Kim Y, Holstein A, Lee D, et al. alimogene Laherparepvec (TVEC) for the Treatment of Advanced Melanoma: A Single-Institution Experience. Ann Surg Oncol (2018) 25 (13):3960-5. doi: 10.1245/s10434-018-6803-0

59. Ozao-Choy J, Lee DJ, Faries MB. Melanoma Vaccines: Mixed Past, Promising Future. Surg Clin North Am (2014) 94:1017. doi: 10.1016/j.suc.2014.07.005

60. Veness MJ, Morgan GJ, Palme CE, Gebski V. Surgery and Adjuvant Radiotherapy in Patients With Cutaneous Head and Neck Squamous Cell Carcinoma Metastatic to Lymph Nodes: Combined Treatment Should be Considered Best Practice. Laryngoscope (2005) 115:870-5. doi: 10.1097/ 01.MLG.0000158349.64337.ED

61. Nehal KS, Bichakjian CK. Update on Keratinocyte Carcinomas. N Engl J Med (2018) 379:363-74. doi: 10.1056/NEJMra1708701

62. DeLeon TT, Salomao MA, Aqel BA, Sonbol MB, Yokoda RT, Ali AH, et al. Pilot Evaluation of PD-1 Inhibition in Metastatic Cancerpatients With a History of Liver Transplantation: The Mayo Clinic Experience. J Gastrointest Oncol (2018) 9:1054-62. doi: 10.21037/jgo.2018.07.05

63. Pantelidou C, Sonzogni O, De Oliveria Taveira M, Mehta AK, Kothari A, Wang D, et al. PARP Inhibitor Efficacy Depends on CD8+ T-Cell Recruitment via Intratumoral STING Pathway Activation in BRCA-Deficient Models of Triple-Negative Breast Cancer. Cancer Discov (2019) 9(6):722-37. doi: 10.1158/2159-8290.CD-18-1218

Conflict of Interest: PB declares advisory board or conference honoraria from Merck, Sanofi-Regeneron, Merck Sharp \& Dohme, Sun Pharma, Angelini, Molteni, Bristol-Myers Squibb, GSK, Nestlè.

The remaining author declares that the research was conducted in the absence of any commercial or financial relationships that could be construed as a potential conflict of interest.

Publisher's Note: All claims expressed in this article are solely those of the authors and do not necessarily represent those of their affiliated organizations, or those of the publisher, the editors and the reviewers. Any product that may be evaluated in this article, or claim that may be made by its manufacturer, is not guaranteed or endorsed by the publisher.

Copyright $\odot 2022$ Alberti and Bossi. This is an open-access article distributed under the terms of the Creative Commons Attribution License (CC BY). The use, distribution or reproduction in other forums is permitted, provided the original author(s) and the copyright owner(s) are credited and that the original publication in this journal is cited, in accordance with accepted academic practice. No use, distribution or reproduction is permitted which does not comply with these terms. 